\title{
Recomendação de Objetos de Aprendizagem utilizando Filtragem Colaborativa baseada em Tendências e em Estilos de Aprendizagem
}

\author{
Janderson J. B. Aguiar', Joseana M. F. R. de Araújo', Evandro de B. Costa ${ }^{2}$ \\ ${ }^{1}$ Universidade Federal de Campina Grande (UFCG), Campina Grande - PB - Brasil \\ ${ }^{2}$ Universidade Federal de Alagoas (UFAL), Maceió - AL - Brasil \\ janderson@copin.ufcg.edu.br, joseana@computacao.ufcg.edu.br, evandro@ic.ufal.br
}

\begin{abstract}
Some researches indicate the importance of considering the particularities of students when designing strategies for Recommender Systems for Learning. In this research, we propose a novel Learning Objects recommendation strategy, a personalized approach based on student Learning Styles and Tendencies-Based Collaborative Filtering - combined with a Genetic Algorithm. An experimental evaluation (using a data set concerning students of Computer Science) indicated that the proposed strategy provided better results when compared to other recommendation approaches.
\end{abstract}

\begin{abstract}
Resumo. Há pesquisas que indicam a importância de considerar particularidades, relativas ao processo de aprendizagem dos estudantes, ao elaborar estratégias para Sistemas de Recomendação de recursos educacionais. Nesta pesquisa, é proposta uma estratégia de recomendação personalizada referente a Objetos de Aprendizagem, com base nos Estilos de Aprendizagem dos estudantes e na Filtragem Colaborativa baseada em Tendências - combinadas por meio de um Algoritmo Genético. Uma avaliação experimental (utilizando um conjunto de dados referentes a estudantes de Computação) indicou que a estratégia proposta proporcionou resultados melhores em comparação a outras abordagens de recomendação.
\end{abstract}

\section{Introdução}

Os Sistemas de Recomendação (SR) podem ser empregados na promoção do processo de aprendizagem, uma vez que, por meio desses sistemas, recursos didáticos são recomendados de maneira personalizada para os estudantes. Entretanto, apesar dos SR serem utilizados satisfatoriamente em uma variedade de domínios, há peculiaridades intrínsecas ao domínio educacional - como aspectos psicopedagógicos - que precisam ser levadas em consideração [Parveen, Jaiswa e Kant 2012] [Costa, Aguiar e Magalhães 2013] [Drachsler et al. 2015] [Aguiar 2017].

Holanda e Tedesco (2017) afirmam que os SR são uma forma de proporcionar personalização em MOOC (Massive Open Online Course - Curso Online Aberto e Massivo) e, além disso, comentam que um dos desafios encontrados na construção e condução de MOOCs é a dificuldade em mapear os perfis dos alunos baseando-se em seu comportamento e interação.

Vários pesquisadores enfatizam que o processo de aprendizagem não é vivenciado por todos os indivíduos da mesma forma, havendo diferentes Estilos de Aprendizagem (EA) [Felder e Silverman 1988] [Kolb 2015] [Klašnja-Milićević et al. 
VII Congresso Brasileiro de Informática na Educação (CBIE 2018)

Anais do XXIX Simpósio Brasileiro de Informática na Educação (SBIE 2018)

2017]. Os EA se referem às maneiras a partir das quais os indivíduos preferem receber e processar informações, podendo-se empregar a identificação desses estilos de forma a propiciar a recomendação de recursos mais efetivos no processo de ensinoaprendizagem [Aguiar et al. 2017].

Sobre as técnicas de SR, a Filtragem Colaborativa (FC) é uma das mais utilizadas [Alyari e Navimipour 2018], inclusive no domínio educacional [Laisa et al. 2018]. As estratégias de FC baseadas em vizinhança (UserKNN e ItemKNN) são bastante populares, sendo cada vez mais utilizadas também as estratégias baseadas em Fatoração de Matrizes (FM). Além dessas, há outras estratégias de FC na literatura, a exemplo do algoritmo TB [Cacheda et al. 2011], que, em vez das semelhanças, considera as diferenças entre usuários/itens, apresentando resultados satisfatórios relativos à acurácia, e destacando-se pela eficiência computacional ${ }^{1}$.

Vários pesquisadores, a exemplo de [Adán-Coello e Yamaguti 2016], [Aggarwal 2016] e [Alyari e Navimipour 2018], comentam a importância de técnicas híbridas de recomendação, combinando duas ou mais técnicas de recomendação diferentes, obtendo as vantagens de cada uma.

Considerando-se o contexto tratado, é proposta neste artigo uma estratégia para SR, no domínio educacional, utilizando os conceitos de EA na construção do perfil dos usuários (estudantes), além de se considerar as tendências de avaliação dos usuários/itens, por meio do algoritmo TB. Os resultados obtidos indicaram vantagem da estratégia híbrida proposta, que também fez uso de um algoritmo genético.

\section{Referencial Teórico}

Em decorrência do crescimento na quantidade de conteúdo disponível na Web, os SR tornaram-se cada vez mais populares e úteis, filtrando os variados dados disponíveis para o usuário, permitindo-lhe encontrar conteúdos apropriados [Aggarwal 2016] [Alyari e Navimipour 2018].

Objetos de Aprendizagem (OA) podem ser definidos como pequenos recursos educacionais digitais que podem ser reutilizados, em diferentes contextos de uso, como apoio à aprendizagem, contribuindo, assim, para a melhoria da educação, tanto em ambientes virtuais quanto presenciais [Wiley 2000 apud Silva e Souza 2017].

Considerando-se a quantidade crescente de $\mathrm{OA}$ disponíveis em vários repositórios existentes, a estratégia proposta neste artigo (Seção 4) visa a propiciar uma seleção personalizada dos OA a serem recomendados. Para isso, é necessário compreender os conceitos das seguintes subseções.

\subsection{Filtragem Colaborativa baseada no FSLSM}

Dentre os modelos de EA, o modelo proposto por Felder e Silverman (1988) — FSLSM (Felder-Silverman Learning Styles Model) — é um dos mais populares [Nascimento et al. 2017], sendo definidas quatro dimensões de EA: (1) Processamento (estilos

\footnotetext{
${ }^{1}$ Cacheda et al. (2011) usaram conjuntos de dados famosos (MovieLens e Netflix) em seus experimentos e apresentaram resultados significativos em relação à acurácia e ao tempo de execução, em comparação a outras técnicas do estado-da-arte (incluindo FM). Destaca-se que o algoritmo TB possui complexidade $\mathrm{O}(m n)$ no treinamento e $\mathrm{O}(1)$ na predição, sendo $m$ o número de usuários e $n$ o número de itens.
} 
VII Congresso Brasileiro de Informática na Educação (CBIE 2018)

Anais do XXIX Simpósio Brasileiro de Informática na Educação (SBIE 2018)

Reflexivo e Ativo); (2) Percepção (estilos Intuitivo e Sensorial); (3) Entrada (estilos Verbal e Visual); e (4) Compreensão (estilos Global e Sequencial).

Aguiar et al. (2017) empregou o uso do FSLSM como forma de calcular a vizinhança em FC. Para isso, usou a Distância Euclidiana para calcular a similaridade entre os estudantes, representados, cada um deles, por um vetor (com valores numéricos relativos às 4 dimensões).

A estratégia de FC baseada em EA utilizada na proposta deste artigo é semelhante a de Aguiar et al. (2017), entretanto, em vez da Distância Euclidiana, foi utilizada a Medida dos Cossenos na estratégia proposta, por ser mais comumente empregada em SR. Tal estratégia de FC baseada em EA é denominada neste artigo como LSBCF (Learning Styles-based Collaborative Filtering). Assim, os EA influenciam a escolha dos $\mathrm{OA}$ a partir das preferências dos usuários com estilos similares aos estilos do estudante-alvo da recomendação.

\subsection{Filtragem Colaborativa baseada em Tendências}

Cacheda et al. (2011) propuseram uma abordagem alternativa à FC, baseando-se em tendências (o qual denominou em seu artigo de algoritmo TB). Esta abordagem parte do princípio que usuários parecidos podem avaliar itens de maneiras diferentes: alguns são inclinados a avaliar com notas altas, deixando notas baixas apenas para os itens realmente ruins; outros costumam avaliar com notas baixas, guardando as maiores notas apenas para os melhores itens.

Em linhas gerais, o algoritmo TB, para prever a avaliação de determinado usuário para determinado item, analisa as avaliações médias do usuário e do item, e as suas tendências, havendo quatro situações possíveis: (I) quando o usuário tende a dar notas acima da avaliação média do item (usuário positivo), e o item tende a ser avaliado acima da média do usuário (bom item); (II) quando o usuário tende a dar notas abaixo da avaliação média do item (usuário negativo), e o item tende a ser avaliado abaixo do nível do usuário (mau item), ou seja, o caso inverso da situação I; (III) quando é um usuário negativo com baixa média, e um bom item com alta média (ou um usuário positivo com alta média, e um mau item com baixa média), isto é, as médias corroboram as tendências; ou, em último caso, (IV) quando é um usuário negativo com alta média, e um bom item com baixa média (ou um usuário positivo com baixa média, e um mau item com alta média), isto é, as médias não corroboram as tendências. Nos casos III e IV, a previsão considera uma constante $\beta$ para controlar a contribuição das médias do item e do usuário na previsão [Cacheda et al. 2011] [Adán-Coello e Yamaguti 2016].

\subsection{Algoritmos Genéticos}

Os Algoritmos Genéticos (GA — do inglês, Genetic Algorithms) são técnicas heurísticas de busca inspiradas pelo processo biológico de evolução natural [Linden 2012], e visam resolver problemas por meio da criação/evolução de populações de indivíduos, que vão se reproduzindo e sendo avaliados.

Em síntese, com base em [Linden 2012] e [Adán-Coello e Yamaguti 2016], um GA pode funcionar da seguinte forma. Inicialmente, são criados indivíduos aleatoriamente (geração inicial) e essa população é avaliada (cada indivíduo representa uma possível solução para o problema). Os indivíduos são submetidos aos operadores genéticos: seleção, recombinação (crossover) e mutação. Os melhores indivíduos são 
VII Congresso Brasileiro de Informática na Educação (CBIE 2018)

Anais do XXIX Simpósio Brasileiro de Informática na Educação (SBIE 2018)

selecionados e o algoritmo é executado enquanto não for encontrada uma solução adequada para o problema (ou até atingir um limite pré-definido de gerações). Cada indivíduo comumente é representado (representação cromossomial) por cadeias de bits. A avaliação de uma população é feita usando uma função de fitness (a função objetivo que se pretende otimizar), útil para selecionar os indivíduos melhor adaptados (as melhores soluções até o momento) — quanto mais adaptado o indivíduo, mais perto se está da solução.

Mais detalhes sobre GA podem ser obtidos no livro de Linden (2012). Comentários sobre GA em SR podem ser encontrados no artigo de Horváth e De Carvalho (2017), uma revisão da literatura relativa a evolutionary computing em SR. Na pesquisa descrita neste artigo, um GA foi usado para combinar os algoritmos de FC descritos nas Subseções 2.1 e 2.2.

Em relação às teorias pedagógicas, ressalta-se que as definições dos EA, segundo Bativa e Stiubiener (2011 apud Aguiar et al. 2017) foram influenciadas pelas teorias de aprendizagem - por exemplo, o construtivismo relaciona-se mais ao estilo ativo de aprendizagem, enquanto o behaviorismo relaciona-se mais ao estilo reflexivo. Além disso, como a denominação da técnica FC sugere, esta pesquisa relaciona-se ao aspecto colaborativo, destacado nas teorias construtivistas de aprendizagem.

\section{Pesquisas Relacionadas}

$\mathrm{Na}$ literatura, há diversas pesquisas relativas a SR utilizando a ideia de FC. Algumas delas, a exemplo de [Adán-Coello e Yamaguti 2016] consideram abordagens híbridas utilizando os conceitos de GA.

Com foco em SR no domínio educacional, há estudos de revisão da literatura em âmbito brasileiro e internacional [Aguiar et al. 2014] [Aguiar et al. 2015] [Drachsler et al. 2015] [Ferreira, Vasconcelos e França 2017] [Klašnja-Milićević et al. 2017] [Laisa et al. 2018] [Nascimento et al. 2017]. Com base nesses estudos, percebe-se que não é novidade usar o conceito de EA para propor estratégias de recomendação, havendo alguns estudos que usam GA. Entretanto, não foram encontradas pesquisas, na área de Informática na Educação, que fizeram uso do algoritmo TB.

Embora também tenham sido lidos outros estudos não elencados nessas revisões citadas, não foram encontrados estudos propondo uma estratégia com todas as características adotadas e descritas neste artigo. Em síntese e de forma comparativa, no Quadro 1, são apresentados alguns dos estudos relacionados encontrados, além da pesquisa proposta neste artigo. A partir do exposto, reforça-se o diferencial da pesquisa realizada em comparação a outras do estado-da-arte.

\section{Estratégia de Recomendação}

A estratégia proposta consiste em recomendar, para determinado estudante-alvo, os OA calculados como os melhores para seu perfil (baseando-se nos EA dos estudantes do sistema e em tendências - algoritmo TB). Para isso, são considerados os dados de cada Perfil de Estudante, descrito a seguir. 
VII Congresso Brasileiro de Informática na Educação (CBIE 2018)

Anais do XXIX Simpósio Brasileiro de Informática na Educação (SBIE 2018)

Quadro 1. Comparação entre pesquisas sobre SR no domínio educacional.

\begin{tabular}{|c|c|c|c|c|c|}
\hline Pesquisa & Recurso recomendado & Estratégia para recomendação & $\begin{array}{l}\text { Uso } \\
\text { de EA }\end{array}$ & $\begin{array}{l}\text { Uso } \\
\text { de TB }\end{array}$ & $\begin{array}{l}\text { Uso } \\
\text { de GA }\end{array}$ \\
\hline Casali et al. (2012) & OA em geral & Baseada em regras & Sim & Não & Não \\
\hline Zaina et al. (2012) & OA em geral & Filtragem baseada em conteúdo & Sim & Não & Não \\
\hline $\begin{array}{l}\text { Silva, Mendes Neto e } \\
\text { Jácome Júnior (2013) }\end{array}$ & OA em geral & $\begin{array}{c}\text { Baseada em agentes para } \\
\text { recomendação sensível ao } \\
\text { contexto (aprendizagem móvel) }\end{array}$ & Não & Não & Sim \\
\hline Vesin et al. (2013) & Links e ações em AVA & $\begin{array}{c}\text { Filtragem baseada em conteúdo, } \\
\text { Baseada em regras }\end{array}$ & Sim & Não & Não \\
\hline $\begin{array}{l}\text { Aguiar, Fechine e } \\
\text { Costa (2015) }\end{array}$ & OA em geral & $\begin{array}{c}\text { Uso de regras baseadas em EA } \\
\text { e popularidade dos itens }\end{array}$ & Sim & Não & Não \\
\hline Dascalu et al. (2015) & $\begin{array}{c}\text { Sugestões e atalhos para } \\
\text { materiais e ferramentas } \\
\text { educacionais }\end{array}$ & $\begin{array}{l}\text { Uso de agente com um } \\
\text { buscador de EA integrado }\end{array}$ & Sim & Não & Não \\
\hline $\begin{array}{l}\text { Adán-Coello e } \\
\text { Yamaguti (2016) }\end{array}$ & Itens em geral $^{2}$ & GA combinando TB e UserKNN & Não & Sim & Sim \\
\hline Dorça et al. (2016) & OA em geral & Uso de regras baseadas em EA & Sim & Não & Não \\
\hline Mendes et al. (2017) & OA em geral & $\begin{array}{l}\text { Clusterização dos OA, uso de } \\
\text { regras baseadas em EA }\end{array}$ & Sim & Não & Não \\
\hline $\begin{array}{c}\text { Christudas, } \\
\text { Kirubakaran e } \\
\text { Thangaiah (2018) }\end{array}$ & OA em geral & $\begin{array}{l}\text { Uso de GA forçando } \\
\text { compatibilidade entre os OA e } \\
\text { os EA dos alunos }\end{array}$ & Sim & Não & Sim \\
\hline PROPOSTA & OA em geral & $\begin{array}{l}\text { GA combinando FC baseada } \\
\text { em EA e em Tendências (TB) }\end{array}$ & Sim & Sim & Sim \\
\hline
\end{tabular}

Cada estudante é representado por um Perfil de Estudante, no qual consta em sua representação uma quádrupla ordenada $\left(d_{1}, d_{2}, d_{3}, d_{4}\right)$, em que: $d_{x} \in \mathbb{N}, 0 \leq d_{x} \leq 100$, $x \in\{1,2,3,4\}$, com cada valor de $x$ referente a uma dimensão do modelo FSLSM (considerando-se a correspondência, entre esses números e as dimensões, apresentada na Subseção 2.1). Uma vez que cada dimensão contempla dois estilos, tem-se que: $d_{l}=0$ representa $0 \%$ para o Estilo Reflexivo e, portanto, $100 \%$ para o Estilo Ativo; $d_{2}=0$ representa $0 \%$ para o Estilo Intuitivo e, portanto, $100 \%$ para o Estilo Sensorial; $d_{3}=0$ representa $0 \%$ para o Estilo Verbal e, portanto, $100 \%$ para o Estilo Visual; $d_{4}=0$ representa $0 \%$ para o Estilo Global e, portanto, 100\% para o Estilo Sequencial.

Além disso, no Perfil de Estudante deve constar a avaliação (feedback avaliativo em uma escala de 1 a 5) para os OA já acessados, sendo esta informação útil para as estratégias colaborativas (tanto para o algoritmo TB quanto para o algoritmo LSBCF).

A partir disso, para se gerar as recomendações, a estratégia proposta utiliza uma abordagem de recomendação híbrida, combinando duas estratégias de recomendação, por meio de um GA. Inicialmente, os OA ainda não acessados pelo estudante-alvo são ordenados de duas formas distintas: com base no algoritmo TB (Subseção 2.2) e com base em EA - algoritmo LSBCF (Subseção 2.1). A partir dessas duas ordenações, é realizada a ordenação final dos $\mathrm{OA}$, de maneira decrescente, com base no Coeficiente de Recomendação $(C)$ do OA, calculado segundo a Equação 1. Por fim, os primeiros OA dessa ordenação final são recomendados ao usuário-alvo.

$$
C(i)=w_{1} \times \frac{1}{P_{L S B C F}(i)}+w_{2} \times \frac{1}{P_{T B}(i)}
$$

\footnotetext{
2 A pesquisa de Adán-Coello e Yamaguti (2016) não enfoca o domínio educacional, mas, dentre os conjuntos de dados utilizados na avaliação experimental, um deles é oriundo de um sistema tutor online.
} 
VII Congresso Brasileiro de Informática na Educação (CBIE 2018)

Anais do XXIX Simpósio Brasileiro de Informática na Educação (SBIE 2018)

Considere em (1) que: $i$ representa o item (OA); $P_{L S B C F}$ representa a posição do OA no ranking relativo ao algoritmo LSBCF; $P_{T B}$ representa a posição do OA no ranking relativo ao algoritmo $\mathrm{TB} ; w_{1}$ e $w_{2}$ são os pesos dados a esses algoritmos, definidos internamente por meio de um GA.

Em relação ao GA utilizado, foram usadas características similares ao GA elaborado por Adán-Coello e Yamaguti (2016). Os indivíduos foram representados como cadeias de 16 bits, sendo os primeiros 8 bits referentes a $w_{1}$ e os últimos 8 referentes a w2. A função de fitness consistiu no cálculo da métrica AUC (Area Under ROC Curve).

\section{Método de Avaliação da Estratégia Proposta}

Para realizar a avaliação da estratégia, foi utilizado o conjunto de dados descrito em [Aguiar, Fechine e Costa 2015], referente a 25 itens (representações dos OA com características diferentes) e 55 usuários (estudantes de Ciência da Computação) - que definiram ratings de 1 a 5 para esses itens, e que responderam ao Index of Learning Styles [Felder e Soloman 1999], instrumento de mensuração referente ao FSLSM.

A partir desse conjunto de dados, foi comparado o desempenho da estratégia híbrida proposta - denominada como TB\&LSBCF_ga - com os desempenhos em separado dos algoritmos TB e LSBCF, da estratégia híbrida sem uso do GA (considerando $\left.w_{1}=w_{2}=1\right)$ - denominada como TB\&LSBCF - e destes outros algoritmos de recomendação (implementações do MyMediaLite [Gantner et al. 2011]): Random (estratégia aleatória, não personalizada), UserKNN e ItemKNN (FC baseada em $k$ usuários/itens, considerados vizinhos mais próximos), WRMF e BPRMF (estratégias usando Fatoração de Matrizes). Para avaliar o desempenho dos algoritmos, foi considerada a relevância das recomendações por meio da métrica AUC. Foi definido $k=15$ para ItemKNN e $k=25$ para os algoritmos UserKNN e LSBCF. O valor da constante $\beta$ do algoritmo TB foi definido como 0,5 .

Para esta análise experimental, foi empregado o método Random Subsampling [Han, Kamber e Pei 2012], sendo separados aleatoriamente, por 100 vezes, os dados em dois conjuntos: um para treinamento, e outro para teste. Embora, tipicamente, atribuemse dois terços dos dados ao conjunto de treinamento, julgou-se pertinente variar, em três níveis, o percentual dos dados utilizado para a base de treinamento. Por isso, foi definido o valor 65\% (aproximadamente dois terços), e a variação de $10 \%$ para mais e para menos $(55 \%$ e $75 \%)$. Para uso do GA, o conjunto de treinamento foi subdividido em treino $(70 \%)$ e validação $(30 \%)$.

\section{Resultados e Discussão}

A fim de verificar o desempenho da estratégia proposta em relação a outros algoritmos, após a execução do experimento planejado, foram gerados intervalos de confiança relativos às médias da métrica de avaliação (AUC) obtidas pelos algoritmos, com nível de significância $(\alpha)$ de 5\%. Além disso, confirmou-se, a partir da ANOVA e do teste de Kruskal-Wallis [Boslaugh e Watters 2008], que os algoritmos obtiveram desempenhos diferentes em relação à métrica AUC.

Na Figura 1, são apresentados os gráficos elaborados relativos aos intervalos de confiança. A partir da análise dos intervalos de confiança (além da execução dos testes T e Mann-Whitney U [Boslaugh e Watters 2008], par a par), percebe-se o melhor 
VII Congresso Brasileiro de Informática na Educação (CBIE 2018)

Anais do XXIX Simpósio Brasileiro de Informática na Educação (SBIE 2018)

desempenho sendo da estratégia híbrida proposta (TB\&LSBCF_ga), seguida da variação dessa estratégia sem uso do algoritmo genético (TB\&LSBCF).

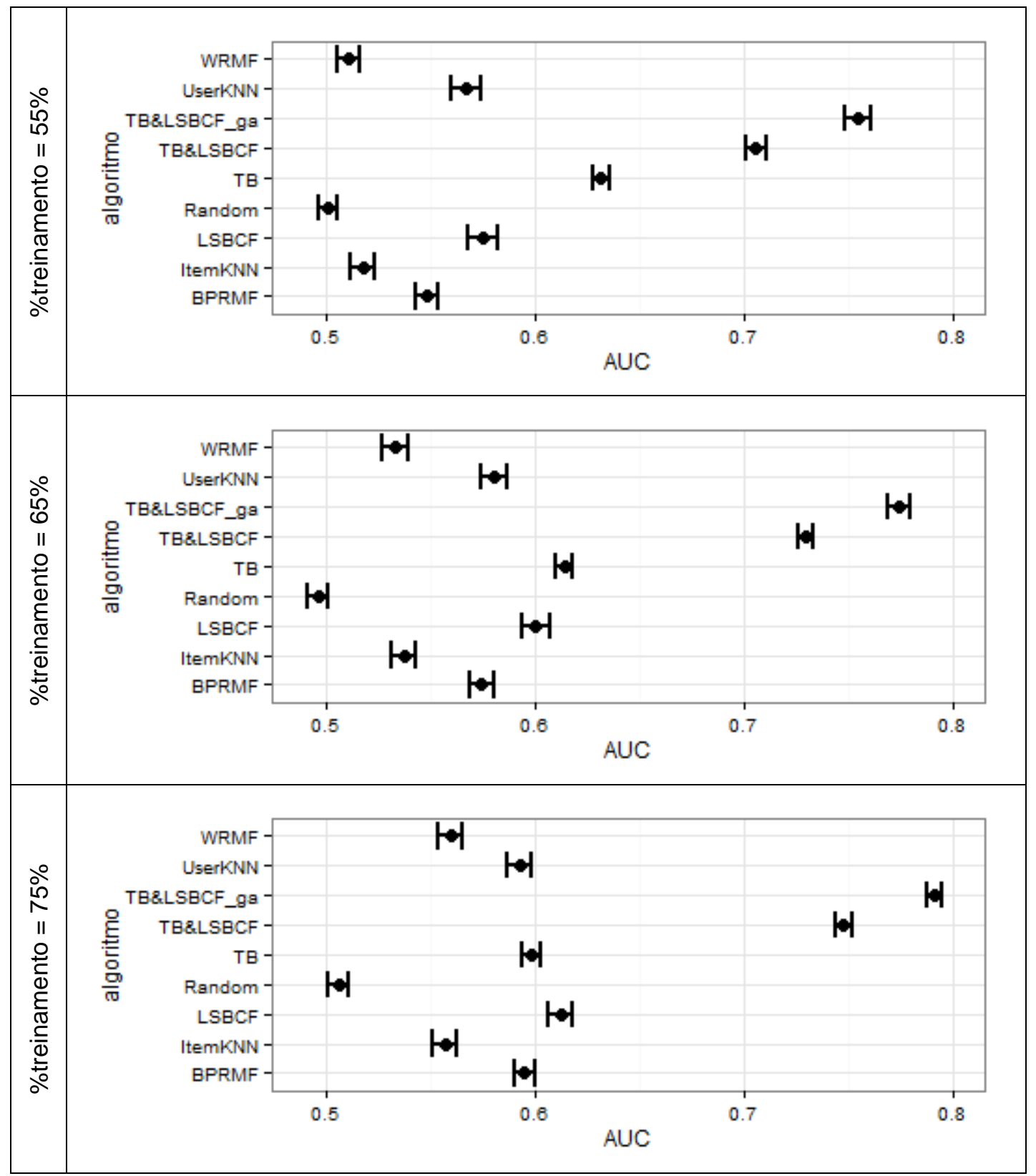

Figura 1. Intervalos de confiança para comparação das abordagens de recomendação (percentual de treinamento variando em $55 \%, 65 \%$ e $75 \%$ ).

É importante ressaltar que os algoritmos que integram a estratégia híbrida proposta, isoladamente (isto é: TB e LSBCF), obtiveram desempenho melhor ou pelo menos similar ao desempenho dos demais algoritmos (Random, UserKNN, ItemKNN, BPRMF e WRMF). Isto reforça a importância dos algoritmos que integram a estratégia híbrida proposta, e, por conseguinte, reforçam a importância das características envoltas a esses algoritmos (os conceitos de EA e Tendências). Em síntese, os resultados indiciam a possibilidade de melhoria das recomendações no domínio educacional a partir da combinação de algoritmos proposta. 
VII Congresso Brasileiro de Informática na Educação (CBIE 2018)

Anais do XXIX Simpósio Brasileiro de Informática na Educação (SBIE 2018)

\section{Considerações Finais}

Embora haja uma quantidade significativa de estudos que abordem SR considerando EA, é escasso o uso da ideia de tendências (algoritmo TB) em SR, especialmente para educação; portanto, a pesquisa em questão visa contribuir com a área de SR ao analisar a aplicação desses conceitos em uma estratégia híbrida de recomendação (aplicando também GA) no domínio educacional.

Apesar de variados estudos em Informática na Educação que utilizam a teoria de EA, há pesquisadores, como Kirschner (2017), que criticam tal utilização, defendendo que faltam, por exemplo, evidências de benefício referente a combinar EA com métodos de ensino. Todavia, como Aguiar et al. (2017) defendem, mesmo sem nítidas evidências relativas à metodologia de ensino, há pesquisas em Informática na Educação mostrando a utilização do conceito de EA como forma de melhorar os SR (como, por exemplo, identificando estudantes com EA similares para definir os vizinhos mais próximos no processo de $\mathrm{FC}$, e/ou resolvendo o problema de cold-start).

Vale ressaltar que a estratégia e os resultados apresentados neste artigo são parte de um projeto em andamento, e pretende-se usar o GA com uma representação cromossomial mais complexa que a que foi apresentada aqui - incluindo, além de $w_{1} \mathrm{e}$ $w_{2}$, o valor da constante $\beta$ do algoritmo TB e ponderações para as dimensões do FSLSM, uma vez que, segundo Aguiar et al. (2017), há indícios que tais dimensões influenciam de modo diferente no processo de recomendação. Visando uma generalização maior dos resultados apresentados, pretende-se também aplicar a estratégia proposta em outros conjuntos de dados. Outro estudo futuro consiste em, além de abordagens de FC, considerar também as características dos itens, fazendo-se uso de regras baseadas em EA, similar a alguns estudos listados no Quadro 1.

Assim como a estratégia de Aguiar, Fechine e Costa (2015), indica-se que, dependendo do ambiente onde for aplicada esta estratégia de recomendação, inicialmente (antes de considerar as abordagens de FC), haja uma filtragem dos OA baseada em seu conteúdo (para, por exemplo, estudantes procurando conteúdo sobre determinada área não receberem recomendação de OA sobre uma área totalmente diferente). Além disso, sugere-se, ao aplicar a estratégia proposta em, por exemplo, um Ambiente Virtual de Aprendizagem (AVA), que as características do perfil do estudante relacionadas a EA sejam atualizadas dinamicamente, como estudado em [Silva e Dorça 2014] e [Falci et al. 2018].

Por fim, considerando-se que há variações quanto ao modo de aprender particular de cada estudante, pretende-se também, com a divulgação desta pesquisa, continuar estimulando demais pesquisadores à realização de estudos considerando aspectos psicopedagógicos em SR na educação, visando à promoção da aprendizagem.

\section{Referências}

Adán-Coello, J. M.; Yamaguti, R. O. (2016). Algoritmo híbrido de recomendação utilizando algoritmo genético. Revista Científica Inovação e Tecnologia, v.2, p. 84-94.

Aggarwal, C. C. (2016). Recommender Systems: The Textbook. 1 ed. Springer International Publishing, 498 p., ISBN: 978-3-319-29659-3.

Aguiar, J. J. B. (2017). Considerando Estilos de Aprendizagem, Emoções e Personalidade em Informática na Educação. Informática na Educação: teoria \& prática, Porto Alegre, v. 20, n. 2, p. $85-102$. 
VII Congresso Brasileiro de Informática na Educação (CBIE 2018)

Anais do XXIX Simpósio Brasileiro de Informática na Educação (SBIE 2018)

Aguiar, J. J. B.; Barbosa, A. F.; Araújo, J. M. F. R.; Costa, E. B. (2017). Um Estudo sobre a Influência das Dimensões do Modelo Felder-Silverman na Recomendação de Recursos Educacionais baseada nos Estilos de Aprendizagem dos Alunos. In: Anais do XXVIII Simpósio Brasileiro de Informática na Educação (SBIE), Recife, p. 1277-1286.

Aguiar, J. J. B.; Fechine, J. M.; Costa, E. (2015). Recomendação de Objetos de Aprendizagem baseada na Popularidade dos Objetos e nos Estilos de Aprendizagem dos Alunos. In: Anais do XXVI Simpósio Brasileiro de Informática na Educação (SBIE), Maceió, p. 1147-1156.

Aguiar, J. J. B.; Santos, S. I. N.; Fechine, J. M.; Costa, E. (2014) Um Mapeamento Sistemático sobre Iniciativas Brasileiras em Sistemas de Recomendação Educacionais. In: Anais do XXV Simpósio Brasileiro de Informática na Educação (SBIE), Dourados, p. 1123-1132.

Aguiar, J. J. B.; Santos, S. I. N.; Fechine, J. M.; Costa, E. (2015). Avaliação de Sistemas de Recomendação Educacionais no Brasil: uma revisão sistemática da literatura. In: Anais do XXVI Simpósio Brasileiro de Informática na Educação (SBIE), Maceió, p. 1255-1264.

Alyari, F.; Navimipour, N. J. (2018). Recommender systems: A systematic review of the state of the art literature and suggestions for future research, Kybernetes, v. 47, n. 5, p. 985-1017.

Boslaugh, S.; Watters, P. A. (2008). Statistics in a Nutshell. O'Reilly Media. ISBN: 9780596510497.

Cacheda, F.; Carneiro, V.; Fernández, D.; Formoso, V. (2011). Comparison of collaborative filtering algorithms: Limitations of current techniques and proposals for scalable, high-performance recommender systems. ACM Transactions on the Web (TWEB), v. 5, n. 1, article 2, p. 1-33.

Casali, A.; Gerling, V.; Deco, C.; Bender, C. (2012). A Recommender System for Learning Objects Personalized Retrieval. In: Santos, O.; Boticario, J. (Org.), Educational Recommender Systems and Technologies: Practices and Challenges, p. 182-210.

Christudas, B. C. L.; Kirubakaran, E.; P. Thangaiah, P. R. J. (2018). An evolutionary approach for personalization of content delivery in e-learning systems based on learner behavior forcing compatibility of learning materials. Telematics and Informatics, v. 35, n. 3, p. 520-533.

Costa, E.; Aguiar, J.; Magalhães, J. (2013). Sistemas de Recomendação de Recursos Educacionais: conceitos, técnicas e aplicações. In: Anais da Jornada de Atualização em Informática na Educação (JAIE/CBIE), p. 57-78.

Dascalu, M-I.; Bodea, C-N.; Moldoveanu, A.; Mohora, A.; Lytras, M.; Pablos, P. O. (2015). A recommender agent based on learning styles for better virtual collaborative learning experiences. Computers in Human Behavior, v. 45, p. 243-253.

Dorça, F. A.; Araújo, R. D.; Carvalho, V. C.; Resende, D. T.; Cattelan, R. G. (2016). An Automatic and Dynamic Approach for Personalized Recommendation of Learning Objects Considering Students Learning Styles: An Experimental Analysis. Informatics in Education, v. 15(1), p. 45-62.

Drachsler, H.; Verbert, K.; Santos, O. C.; Manouselis, N. (2015). Panorama of Recommender Systems to Support Learning. In: Ricci, F.; Rokach, L.; Shapira, B. (Org.), Recommender Systems Handbook, p. 421-451.

Falci, S. H.; Vivas, A.; Assis, L.; Pitangui, C.; de Carvalho, L. L.; Dorça, F. (2018). Deteç̧ão de Estilos de Aprendizagem utilizando Lógica Fuzzy e Categorização de Reforços. In: Anais do Congresso Internacional de Educação e Tecnologias (CIET:EnPED), [S.1.].

Felder, R. M.; Silverman, L. K. (1988). Learning and Teaching Styles in Engineering Education. Journal of Engineering Education, v. 78, n. 7, p. 674-681.

Felder, R. M.; Soloman, B. A. (1999). Index of Learning Styles (ILS). Disponível em: $<$ http://www4.ncsu.edu/unity/lockers/users/f/felder/public/ILSpage.html $>$. Acesso em: 02 jul. 2018.

Ferreira, V. A. S.; Vasconcelos, G. C.; França, R. S. (2017). Mapeamento Sistemático sobre Sistemas de Recomendações Educacionais. In: Anais do XXVIII Simpósio Brasileiro de Informática na Educação (SBIE), Recife, p. 253-262. 
VII Congresso Brasileiro de Informática na Educação (CBIE 2018)

Anais do XXIX Simpósio Brasileiro de Informática na Educação (SBIE 2018)

Gantner, Z.; Rendle, S.; Freudenthaler, C.; Schmidt-Thieme, L. (2011). MyMediaLite: A Free Recommender System Library. In: Proceedings of the Fifth ACM Conference on Recommender Systems (RecSys 2011). Chicago, USA, p. 305-308.

Han, J.; Kamber, M.; Pei, J. (2012). 8 - Classification: Basic Concepts. In: Data Mining: Concepts and Techniques (Third Edition) - A volume in The Morgan Kaufmann Series in Data Management Systems, p. 327-391, ISBN: 978-0-12-381479-1.

Holanda, A. C.; Tedesco, P. (2017). MOOCs e Colaboração: definição, desafios, tendências e perspectivas. In: Anais do XXVIII Simpósio Brasileiro de Informática na Educação (SBIE), Recife, p. 243-252.

Horváth, T.; De Carvalho, A. C. P. L. F. (2017). Evolutionary computing in recommender systems: a review of recent research. Natural Computing, v. 16, p. 441-462.

Kirschner, P. A. (2017). Stop propagating the learning styles myth. Computers \& Education, v. 106(3), p. 166-171.

Klašnja-Milićević, A.; Vesin, B.; Ivanović, M.; Budimac, Z.; Jain, L. C. (2017). E-Learning Systems: Intelligent Techniques for Personalization. Springer, ISBN: 978-3-319-41163-7.

Kolb, D. A. (2015). Experiential learning: experience as the source of learning and development (2nd ed.). Upper Saddle River, NJ: Pearson Education. ISBN: 978-0-13-389240-6.

Laisa, J.; Medeiros, T.; Aranha, E.; Silva, T. R. (2018). Uma Revisão Sistemática da Literatura sobre Sistemas de Recomendação Educacional. In: Anais do IX Computer on the Beach, Florianópolis, p. 751-760.

Linden, R. (2012). Algoritmos Genéticos. 3 ed. Rio de Janeiro: Ciência Moderna. ISBN: 9788539901951.

Mendes, M. M.; Carvalho, V. C.; Dorça, F. A.; Araújo, R. D.; Cattelan, R. G. (2017). Agrupamento e Recomendação de Objetos de Aprendizagem no Padrão IEEE-LOM Considerando Estilos de Aprendizagem. In: Anais do XXVIII Simpósio Brasileiro de Informática na Educação (SBIE), Recife, p. 1217-1226.

Nascimento, P.; Barreto, R.; Primo, T.; Gusmão, T.; Oliveira, E. T. H. (2017). Recomendação de Objetos de Aprendizagem baseada em Modelos de Estilos de Aprendizagem: Uma Revisão Sistemática da Literatura. In: Anais do XXVIII Simpósio Brasileiro de Informática na Educação (SBIE), Recife, p. 213-222.

Parveen, R.; Jaiswa, A. K.; Kant, V. (2012). E-Learning Recommendation Systems - A Survey. International Journal of Engineering Research and Development, v. 4, p. 10-12.

Silva, D. H.; Dorça, F. A. (2014). An Automatic Approach for Customization of Teaching Process Based on Learning Styles in Adaptive and Intelligent Learning Systems. Brazilian Journal of Computers in Education (RBIE), v. 22, n. 2, p. 1-15.

Silva, J. W. F.; Souza, C. T. (2017). Repositórios de Objetos de Aprendizagem: características; classificações; limitações e tendências. In: Anais do XXVIII Simpósio Brasileiro de Informática na Educação (SBIE), Recife, p. 61-70.

Silva, L. C.; Mendes Neto, F. M.; Jácome Júnior, L. (2013). MobiLE: A Mobile Learning Multi Agent Environment Based in Genetic Algorithm to Support Ubiquitous Learning. Brazilian Journal of Computers in Education (RBIE), v. 21, n. 1, p. 62-75.

Vesin, B.; Klasnja-Milicevic, A.; Ivanovic, M., Budimac, Z. (2013). Applying Recommender Systems and Adaptive Hypermedia for e-Learning Personalization. Computing and Informatics, v. 32, p. 629-659.

Zaina, L. A.; Bressan, G.; Cardieri, M. A. A.; Rodrigues Junior, J. F. (2012). e-LORS: Learning Objects Recommendation Approach. Brazilian Journal of Computers in Education (RBIE), v. 20, n. 1, p. 4-16. 